\title{
Editorial
}

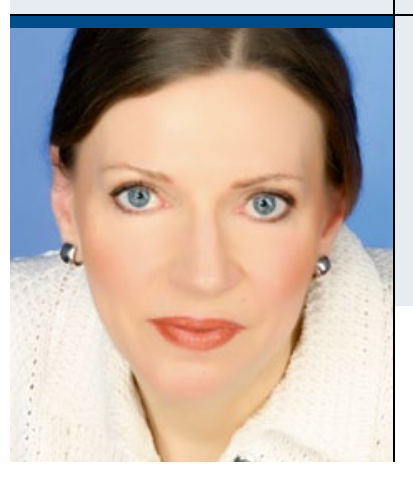

„Wir Urologen dürfen uns die Kompetenz auf dem Gebiet der

Urindiagnostik und Mikrobiologie nicht wegnehmen lassen."

Dr. med. Elke Heßdörfer

Fachärztin für Urologie, Blasenzentrum Westend, Berlin

\section{Flagge zeigen!}

D ie aktuelle URO-NEWS-Sommerausgabe hat den Schwerpunkt Mikrobiologie. Diese ist eigentlich unser tägliches Brot, vor allem was die Behandlung von Harnwegsinfekten angeht. Allerdings drängt sich mir in letzter Zeit immer mehr der Eindruck auf, dass Harnwegsinfekte eher lästiger Ballast sind, sofern es sich um weibliche Patienten (immerhin das Gros der Betroffenen) handelt. Sie kommen immer im unpassenden Moment und bringen die Sprechstunde durcheinander; nicht selten wird rasch ein Rezept ausgestellt und die weitere Behandlung an das Praxispersonal delegiert. Was ist schon ein banaler Harnwegsinfekt im Vergleich zu ernsthaft erkrankten Tumorpatienten! Es bleibt nur die Frage, wie lange wir uns als Fachgruppe diese Denkweise noch leisten können. Wir sollten vielmehr klar Flagge zeigen auf diesem Gebiet. Das fängt schon mit der Umsetzung der (ungeliebten) Leitlinien an. Der reflektorische Griff zum Gyrasehemmer-Rezept für die weiblichen Patienten sollte wenigstens bei uns Urologen der Vergangenheit angehören. Apropos Vergangenheit: Harnröhrenkalibrierungen scheinen vergessen, wenngleich von unseren Altvorderen schon Ende des 19. Jahrhunderts der Zusammenhang zwischen rezidivierenden Harnwegsinfekten und Meatusengen erkannt worden war. Katheterurine erfreuen sich, seit sie nicht mehr vergütet werden, auch nur geringer Beliebtheit - ich frage mich, welche therapeutischen Konsequenzen Mittelstrahl- oder besser gesagt Spontanurine mit mehreren Keimen haben - hoffentlich nicht die Konsequenz eines „one fits all“-Breitbandantibiotikums!

So banal die unkomplizierte Zystitis auch ist, so sehr leiden Frauen darunter, insbesondere diejenigen mit rezidivierenden Zystitiden. Rechtzeitige Aufklärung und Information zu Prophylaxemaßnahmen wären für die Betroffenen vonnöten und würden dankbar angenommen werden. Ist Geschlechtsverkehr ein Hauptauslöser, sollte natürlich der Partner untersucht werden. Dabei ist nicht nur an Chlamydien oder Mykoplasmen zu denken, sondern vielmehr an ganz normale uropathogene Keime, die sich im Ejakulat oder auf dem Penis tummeln können - eine Urinuntersuchung ist da wenig hilfreich. Leider gibt es keine evidenzbasierten Daten über die Häufigkeit derartiger stiller Infektionen, die gar nicht so selten sind, wenn man danach sucht („,expert opinion“). Ob die Männer die Bakterien von ihren Partnerinnen bekommen haben oder umgekehrt ist völlig unerforscht. Und Frauen mit steriler Urinkultur, die über Zystitisoder Urethritissymptome klagen, müssen nicht gleich eine Somatisierungsstörung oder gar interstitielle Zystitis haben: Häufig sind Anaerobier wie Gardnerella oder Atopobium die Ursache, wie Prof. Blenk im Beitrag "Partnertherapie bei Gardnerella-Infektionen" auf Seite 28 veranschaulicht.

Zudem wird momentan diskutiert, ob das Syndrom der überaktiven Blase möglicherweise eng mit einer Urozystitis in Zusammenhang zu sehen ist und in der $\mathrm{Zu}$ kunft mit Antibiotika zu behandeln ist. Circa $30 \%$ der OAB-Patienten haben eine Bakteriurie, aber auch bei steriler Urikultur lassen sich inzwischen im Urin Entzündungsmarker wie Lactoferrin und IL-6 nachweisen. Durch eine chronische Urozystitis hochregulierte Rezeptoren hätten dann eine überaktive Blase zur Folge.

Kurz und gut: Wir Urologen dürfen uns die Kompetenz auf dem Gebiet der Urindiagnostik und Mikrobiologie und somit der Blase nicht wegnehmen lassen. Wir müssen aber auch am Ball bleiben.

In diesem Sinne eine schöne Ferienzeit wünscht Ihnen
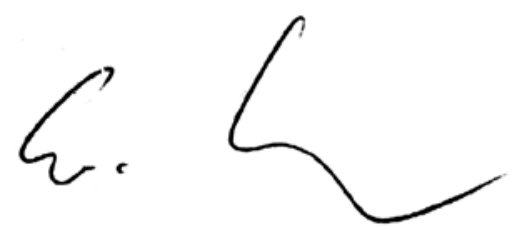